\title{
ENABLING STAR FORMATION VIA SPONTANEOUS MOLECULAR DIPOLE ORIENTATION IN ICY SOLIDS
}

\author{
Alexander Rosu-Finsen ${ }^{1}$, Jérôme Lasne ${ }^{1,2}$, Andrew Cassidy $^{3}$, Martin R. S. McCoustra ${ }^{1}$, And David Field ${ }^{3,4}$ \\ ${ }^{1}$ Institute of Chemical Sciences, Heriot-Watt University, Riccarton, EH14 4AS Edinburgh, UK \\ ${ }^{2}$ Laboratoire Interuniversitaire des Systèmes Atmosphériques (LISA), CNRS UMR 7583, Université Paris-Est Créteil, Université Paris Diderot, \\ Faculté des Sciences et Technologie, 61 avenue du Général de Gaulle, 94010 Créteil Cedex, France \\ ${ }^{3}$ ISA, Department of Physics and Astronomy, Aarhus University, DK-8000 Aarhus C, Denmark; dfield@phys.au.dk \\ Received 2016 May 18; revised 2016 September 26; accepted 2016 September 27; published 2016 November 10
}

\begin{abstract}
It is shown here how new experimental data, for the electrical properties of solid $\mathrm{CO}$, can be used to fill important gaps in our understanding of the evolution of prestellar cores. Dust grains with a mantle of CO lead to a reduction in the degree of ionization in these cores by a factor of between 5 and 6 . The lifetimes for expulsion of magnetic fields from cores, a process generally necessary for gravitational collapse, are reduced from current estimates of several megayears, by a similar factor. This removes a major inconsistency, since lifetimes now tally with typical ages of prestellar cores of a few hundred thousand to $10^{6} \mathrm{yr}$, derived from observations. With the reduced timescales, cores also escape disruption by Galactic supernova remnants. Our results provide a natural mechanism for the generation of so-called magnetically supercritical cores, in which the magnetic field alone cannot prevent gravitational collapse. In addition, we find a minimum value for the density of prestellar cores of $\geqslant(1.1 \pm 0.1)$ $\times 10^{4} \mathrm{H}_{2} \mathrm{~cm}^{-3}$, in agreement with observations.
\end{abstract}

Key words: ISM: clouds - magnetic fields - methods: laboratory: solid state - molecular processes - stars: formation

\section{INTRODUCTION}

Key to understanding the evolution of galaxies is knowledge of how the interstellar environment controls star formation. Major hurdles exist in this field, in particular with regard to the earliest stages of stellar evolution, prior to the gravitational collapse of prestellar cores for low-mass stars (Nielbock et al. 2012). First, one may compare the lifetimes of cores with the time for expulsion of the magnetic field through ambipolar diffusion. Core lifetimes deduced from numerous data (e.g., optical, submillimeter, chemical age; WardThompson et al. 2007; Enoch et al. 2008; Evans et al. 2009; Pagani et al. 2013; Brünken et al. 2014; Könyves et al. 2015) are subject to considerable uncertainty. Estimates may extend up to $>3 \times 10^{6} \mathrm{yr}$, for low number density cores, down to a few times $10^{5} \mathrm{yr}$ (Maret et al. 2013) or as low as $10^{5} \mathrm{yr}$ (Lippok 2014). The majority of estimates, however, tend to lie between 250,000 to $10^{6} \mathrm{yr}$, whereas expulsion of the magnetic field through ambipolar diffusion, the rate determining step in low-mass protostar formation, has been estimated to have a characteristic timescale, $\tau$, of $\lesssim 3 \times 10^{6}$ yr (Draine 2011). Second, almost the entire spiral arm star-forming volume of the Galaxy is overrun by overlapping remnants of supernova explosions (SNe) in $\tau_{\mathrm{SNR}}=2 \pm 0.5 \mathrm{Myr}$ (Tielens 2006; Draine 2011; Korolev et al. 2015), given that SNe occur at the rate of $2 \pm 0.5$ per century (Diehl et al. 2006) and each SN perturbs a region in a cloudy interstellar medium (ISM) of radial dimension $\sim 60 \mathrm{pc}$ (Korolev et al. 2015). The upshot is that the quiescent conditions necessary for gravitational collapse cannot in general be met or that the timescale for the establishment of these conditions is significantly extended. Third, there is the long-standing problem (e.g., Banerjee \& Körtgen 2015) of the mechanism of generation of magnetically supercritical regions. Magnetic fields, if sufficiently strong, prevent the collapse of cores of gas by exerting magnetic

\footnotetext{
${ }^{4}$ Corresponding author.
}

pressure. Thus, the mass to magnetic flux ratio must be greater than a certain value, which may be estimated to be $5.6 \times 10^{7}$ $\mathrm{T}^{-1} \mathrm{~m}^{2} \mathrm{~kg}$ (Draine 2011), for the core to become supercritical and for collapse to take place. Can ambipolar diffusion take place sufficiently rapidly for this condition to be satisfied within the effective lifetime of a quiescent core? Fourth, there is the question of a minimum value for the average density of a prestellar core (Lada et al. 2010; Parmentier et al. 2011): is there such a minimum, and, if so, can we rationalize the value that this density takes?

We show here that the four problems outlined above are closely connected and all four can be solved simultaneously using a potent combination of observations of the strong depletion onto dust grains of gas-phase $\mathrm{CO}$ in prestellar cores (e.g., Bergin \& Tafalla 2007; Ford \& Shirley 2011; Parise et al. 2011; Pagani et al. 2012) and recent laboratory data (RosuFinsen et al. 2016) for the properties of solid CO. The crux of the present work is that laboratory data demonstrate that solid $\mathrm{CO}$, through its spontaneously electrical ("spontelectric") nature (Balog et al. 2009; Field et al. 2013), provides an efficient mechanism for the reduction of ionization in cores. This results in a decrease of the lifetime for ambipolar diffusion, $\tau$, through a factor of $\sim 5.5$, such that (i) $\tau$ matches the range of lifetimes of prestellar cores deduced from observations, (ii) $\tau$ becomes less than $\tau_{\mathrm{SNR}}$, (iii) a natural route is provided to magnetic supercriticality, and (iv) it is found that the minimum average density of a core must be $\geqslant(1.1 \pm 0.1) \times 10^{4} \mathrm{H}_{2} \mathrm{~cm}^{-3}$, in agreement with observations (Lada et al. 2010; Parmentier et al. 2011).

There have been extensive observations of molecular clouds, which relate their size, contained mass, and magnetic fields through power-law relationships with number density (Crutcher et al. 2010; Draine 2011; Crutcher 2012; Roy et al. 2014; Li et al. 2015; Tritsis et al. 2015). Relationships given in Draine (2011) and Crutcher et al. (2010) yield the general result that for low-mass, cold, quiescent prestellar cores, the ratio of mass to magnetic flux is less than the critical 
Table 1

Parameters and Expressions Used in the Calculation of the Polarization Charge on an Isolated Grain (Equation (1)) and of the Ambipolar Diffusion Times in a Prestellar Core $\tau$ and $\tau_{\mathrm{s}}$, the Latter in the Presence of the Spontelectric Effect

\begin{tabular}{|c|c|c|}
\hline Symbol & Description & Value Used \\
\hline $\bar{\phi}$ & $\begin{array}{l}\text { Number of volts added per ML } \\
\text { of CO }\end{array}$ & $(6.7 \pm 0.5) \times 10^{-3} \mathrm{~V} \mathrm{ML}^{-1}$ \\
\hline$q_{p}$ & $\begin{array}{l}\text { Polarization charge on an } \\
\text { isolated grain }\end{array}$ & $5.5 \pm 0.4$ \\
\hline$a_{b}$ & Bare grain radius & $0.1 \mu \mathrm{m}$ \\
\hline$a$ & $\begin{array}{l}\text { Grain radius with } \mathrm{CO} \text { and water } \\
\text { ice mantle }\end{array}$ & $0.115 \mu \mathrm{m}$ \\
\hline$n_{\mathrm{ML}}$ & Number of $\mathrm{ML}$ of $\mathrm{CO}$ on grain & 10.2 \\
\hline$\delta$ & Density of grain material & $2000 \mathrm{~kg} \mathrm{~m}^{-3}$ \\
\hline$\xi$ & Grain-to-gas-mass ratio & 0.013 \\
\hline$n_{g}$ & Number of grains $\mathrm{m}^{-3}$ & $2.1 n_{\mathrm{H} 2} \cdot m_{\mathrm{H}} \xi / \pi a_{b}^{3} \delta$ \\
\hline$\alpha$ & $\begin{array}{l}\text { Degree of ionization of the } \\
\text { medium }\end{array}$ & $6.7 \times 10^{-3} \chi\left\langle n_{\mathrm{H}}\right\rangle^{1 / 2}$ \\
\hline$\chi$ & $\begin{array}{l}\text { Factor of reduction of ion } \\
\text { concentration }\end{array}$ & 0.182 \\
\hline$r_{m}$ & $\begin{array}{l}\text { The maximum radius of } \\
\text { the core }\end{array}$ & $2.39 \times 10^{24} n_{\mathrm{H}}^{-0.81}$ \\
\hline$\left\langle n_{\mathrm{H}}\right\rangle$ & $\mathrm{H}$ nuclei $\mathrm{m}^{-3}$ & $1.25 \times 10^{30} r_{m}^{-1.23}$ \\
\hline$T$ & Kinetic temperature & $10 \mathrm{~K}$ \\
\hline
\end{tabular}

Note. Further details may be found in Rosu-Finsen et al. (2016). For error estimates, see the caption to Figure 1.

value, that is, cores are in general magnetically subcritical. Given the general primacy of the initial magnetic field in supporting low-mass cores against gravitational collapse, this in turn indicates the necessity of the expulsion of the field for collapse to proceed (Crutcher et al. 2010). Note that magnetic fields here, and from hereon, refer to initial magnetic conditions, that is, before any expulsion of the field has taken place.

To illustrate subcriticality in a specific case, a B68 avatar is used, which has the same dimension and mass as present-day B68 (Nielbock et al. 2012; Roy et al. 2014), but contains the original magnetic field that its dimension and mass require (Crutcher et al. 2010; Draine 2011). Given a radius of $0.18 \mathrm{pc}$ and a total mass of $3.1 M_{\odot}$ (Nielbock et al. 2012), it may be inferred that B68 had a pristine magnetic field of $1.29 \times 10^{-8}$ $\mathrm{T}$, as detailed in Section 3.1 below. It follows that the ratio of mass to magnetic flux was originally $2.6 \times 10^{7} \mathrm{~T}^{-1} \mathrm{~m}^{2} \mathrm{~kg}$, less than the critical value for support against gravitational collapse by a factor of $\sim 2$, given a poloidal field. Thus, B68 was originally magnetically subcritical and was not subject to collapse. In the evolved state of B68, as of now, the observed magnetic field (Kandori et al. 2009) is $\sim 0.2 \times 10^{-8} \mathrm{~T}$, showing that B68 is currently hovering on the border between magnetically sub- and supercritical.

In connection with the above discussion, models based on the notion that super-Alfvénic turbulence controls star formation in prestellar cores, without transition into a subsequent quiescent phase (Banerjee \& Körtgen 2015), are in conflict with observations both that line widths are narrow in these cores, of typically $0.1-0.2 \mathrm{~km} \mathrm{~s}^{-1}$ (Caselli 2011; Bergin \& Tafalla 2007), and of CO ice formation (Section 2), since super-Alfvénic turbulence would shatter $\mathrm{CO}$ ices (Tielens 2006). From hereon we make the simplifying approximation that we may ignore any contribution from turbulence. In support of this, accumulating observational evidence
(Sadavoy et al. 2015) shows that cores must first dissipate their turbulence before the onset of $\mathrm{CO}$ depletion, the topic to which we now turn.

\section{SOLID CO ON GRAINS: ELECTRICAL PROPERTIES}

As noted, numerous observations have shown that prestellar cores are characterized by the disappearance of $\mathrm{CO}$ from the gas phase and its reappearance in the solid form. We now describe how the expulsion of the magnetic field is significantly hastened through the presence of a spontelectric film of solid $\mathrm{CO}$ on dust grains in the core.

When dipolar species, such as $\mathrm{CO}$, are allowed to deposit in the laboratory on a cold surface under vacuum to form a layer of approximately 4-5 monolayers (ML), the molecules may spontaneously orient throughout the layer such that their positive or negative ends protrude from the surface, creating a polarization potential (Balog et al. 2009; Cassidy et al. 2012; Field et al. 2013; Lasne et al. 2015; Rosu-Finsen et al. 2016). We have developed a semi-empirical description of this socalled spontelectric effect, which describes major features of the phenomenon. This description is based on a physical model that invokes spontaneous orientation of the dipolar molecules of which the film is composed. The analysis, described in detail in Field et al. (2013), is remarkably successful in describing important characteristics of spontelectrics, such as the deposition temperature dependence of the surface polarization charge, including the counterintuitive behavior of solid methyl formate (Plekan et al. 2011). In the case of CO, the positive oxygen end protrudes on average (Collings et al. 2014). The spontelectric effect is exemplified by a powerful spontaneous electric field in the film. It was first discovered for nitrous oxide using a direct measurement of surface potential, employing high-resolution, low-energy electron beams (Balog et al. 2009). Studies have now been extended to $\mathrm{CO}$ using RAIR (reflection-absorption infrared) spectroscopy (Rosu-Finsen et al. 2016). RAIR spectra were recorded of CO laid down to a thickness of $20 \mathrm{ML}$, dosed at 21,22 , and $24 \mathrm{~K}$, on $50 \mathrm{ML}$ of compact amorphous solid water $\left(c_{\mathrm{ASW}}\right)$ formed by dosing at $110 \mathrm{~K}$, in order to reproduce as far as possible the nature of ices on grains in a prestellar core. The presence of spontaneous electric fields in the film of $\mathrm{CO}$ was demonstrated by measurements of Stark frequency shifts in RAIR spectra, the values of which are film deposition temperature dependent. These observations were used to derive the electric field in the film, yielding a temperature-averaged surface potential, $\phi$, of $6.7 \pm 0.5 \mathrm{mV}$ per ML of CO (RosuFinsen et al. 2016). We note that experimental observations show that the surface potential for any spontelectric material depends on the temperature of the substrate at the moment of deposition, which is $\leqslant 26 \mathrm{~K}$ in the ISM. The subsequent cooling of the substrate to $10 \mathrm{~K}$ in the ISM has no effect on the surface potential.

The spontelectric effect gives rise to a positive polarization charge, $q_{p}$, on the surface of an isolated grain, which may be directly related to the value of $\phi$ by

$$
q_{p}=4 \pi \epsilon_{0} a n_{\mathrm{ML}} \phi
$$

where $a$ is the grain radius and $n_{\mathrm{ML}}$ is the number of ML of CO on any grain, which we note is independent of the value of $a$. Integration of the grain size distribution (e.g., Mathis et al. 1977) shows that a single value may be assigned of $a_{b}=0.1 \mu \mathrm{m}$ for the bare grain, as representative of grain sizes 
in the ISM. Thus, $a$, on inclusion of $40 \mathrm{ML}$ of $c_{\mathrm{ASW}}$ of layer spacing $0.318 \mathrm{~nm}$ and $n_{\mathrm{ML}}$ of CO ice of spacing $0.339 \mathrm{~nm}$ (Ford \& Shirley 2011; Rosu-Finsen et al. 2016), becomes $0.112+3.39 \times 10^{-4} n_{\mathrm{ML}} \mu \mathrm{m}$. CO has been observed to be depleted by factors of up to several hundred in cores (e.g., Pagani et al. 2012). Using the values shown in Table 1, there are $10.2 \mathrm{ML}$ of $\mathrm{CO}$ on a dust grain, given $\mathrm{CO} / \mathrm{H}_{2}=10^{-4}$ before freeze-out, with an associated time of formation of typically $10^{5} \mathrm{yr}$ for $95 \%$ depletion of $\mathrm{CO}$ from the gas phase. This yields $a=0.115 \mu \mathrm{m}$ for the B68 avatar (Table 1).

The condition that must be maintained in the ISM is that there is no gradient in electrochemical potential between the electrons in the gas phase and those on the grains. This condition is equivalent to the statement that an equilibrium charge is acquired by any grain. In the absence of spontelectric $\mathrm{CO}$, it turns out that this condition is ensured by the presence of an approximately single negative charge on a representative grain, in the shielded ISM. Due to local electric fields created through electron depletion, positive ions follow the electrons to grain surfaces, thus preserving electroneutrality in the plasma. On the grain surface, positive ions recombine efficiently with the mobile surface electron (Umebayashi \& Nakano 1980; Draine \& Sutin 1987; Field 2000; Rosu-Finsen et al. 2016). Further electrons now flow to the grain surfaces. Ionization in the plasma at some fixed level, discussed below, is maintained by cosmic-ray events. In this connection, the dominance of the surface recombination of ions and electrons in determining the degree of ionization of the medium was first put forward in Umebayahsi \& Nakano (1980) and demonstrated conclusively in Draine \& Sutin (1987). As discussed in detail in RosuFinsen et al. (2016), in the absence of the spontelectric effect of $\mathrm{CO}$ on grains, grain surface recombination dominates the gasphase mechanism by a factor of $\sim 7.5$ in removal of ionization from the gas phase and by a factor of $\sim 41$ in the presence of spontelectric grains.

As $\mathrm{CO}$ deposits, any grain surface becomes less negative due to the spontelectric effect, and its electrochemical potential is effectively offset from that of the plasma. The electrochemical potential of the grain surface therefore no longer equals that of the gas-phase electrons, the system moving away from the nonspontelectric equilibrium. This causes a net flow of electrons toward the grain, down the electrochemical potential gradient. This in turn causes more positive ions to deposit on the dust grains and hence increases recombination rates. In a fully fledged CO layer of 10.2 ML, a new equilibrium between cosmic-ray ionization and recombination on dust grains is now established by maintaining the equality of the electrochemical potential of gas-phase and grain-surface electrons. This may be satisfied by a reduction in the degree of ionization in the plasma through a factor of $q_{p}$, Equation (1), as outlined below, on the assumption that the surface recombination mechanism is dominant in determining the degree of ionization in the shielded ISM (Rosu-Finsen et al. 2016), as mentioned above.

The factor of $q_{p}$ reduction in ionization may readily be appreciated by recognizing that the system must adjust to an ion-electron surface recombination rate proportional to the product of the surface concentration of electrons and ions, that is, $q_{p}^{2}$. To retain equilibrium, a corresponding gas-phase reduction in electron and ion concentration must follow, each through a factor $q_{p}$. In addition, it was shown in Rosu-Finsen et al. (2016) that both a phenomenological model, based on electron mobility on grain surfaces (Field 2000), and the theoretical description of Draine \& Sutin (1987) yield a degree of ionization $\alpha=3 \times 10^{-8}$ in the non-spontelectric case, given an assumed cosmic-ray ionization rate of $3 \times 10^{-17} \mathrm{~s}^{-1}$. In the present case, given that $\alpha=6.7 \times 10^{-3}\left\langle n_{\mathrm{H}}\right\rangle^{-1 / 2}$ (Draine 2011, chap. 16), the degree of ionization for our B68 avatar is $\sim 3 \times 10^{-8}$, using $\left\langle n_{\mathrm{H}}\right\rangle=4.46 \times 10^{10} \mathrm{~m}^{-3}$ for B68 (see below). In the presence of spontelectric grains $\alpha$ is reduced by a factor equal to the number of polarization charges, $q_{p}$, on an isolated grain. With the value of $\phi$ of $(6.7 \pm 0.5)$ $\times 10^{-3} \mathrm{~V} \mathrm{ML}^{-1}$ (Table 1), Equation (1) shows that the resulting polarization charge contribution to an isolated grain would be equivalent to $q_{p}=+5.5 \pm 0.4$ charges (Table 1 ). Hence, the degree of ionization is equal to $6.7 \times 10^{-3} n_{\mathrm{H}}^{-1 / 2} / q_{p}$, yielding a value of $\alpha=5.8 \times 10^{-9}$, substantially in agreement with an observationally based figure of $5 \times 10^{-9}$ relative to $\mathrm{H}$ nuclei for B68 (Maret \& Bergin 2007). In this connection, the rate of reionization of the medium is very rapid compared to the rate at which ions are lost in the process of expulsion of the magnetic field.

We note that in the relevant plasma environment, the rate of bombardment by electrons and ions is very much greater than the rate of accumulation of $\mathrm{CO}$ layers and of the corresponding spontelectric polarization charge (Rosu-Finsen et al. 2016). Therefore, grains in space cannot achieve an average polarization charge of 5.5 but oscillate between a single positive and negative charge and neutral in response to the arrival of electrons and ions on the grain surface. In our estimation of $q_{p}$, it is assumed that the $\mathrm{CO}$ ice is pure $\mathrm{CO}$; it may be diluted to some extent by $\mathrm{N}_{2}$ (Pagani et al. 2012). This is ignored here, since experiments have shown that weak dilution of spontelectrics has only a small effect on the resulting polarization charge (Cassidy et al. 2014).

\section{DIPOLE-ORIENTED CO AND THE TIMESCALE OF AMBIPOLAR DIFFUSION}

Our purpose now is to calculate the extent of enhancement of the rate of expulsion of the magnetic field that accompanies the reduction in the degree of ionization, where this is due to the spontelectric effect of the CO-coated grains. It is a good approximation to assume that the magnetic field is frozen into the plasma (Draine 2011) and is so strongly coupled to charged particles that their expulsion, through a magnetic pressure gradient, also expels the magnetic field. In a static cloud, the timescale, $\tau$, of ambipolar diffusion to remove ions and thereby the magnetic field from a prestellar core may be represented as the radius of the core divided by the average relative velocity of ions and neutrals. The latter is given by the balance between the mechanical friction between ions and neutrals and the gradient of magnetic pressure $\propto d / d r B_{0}^{2}$, where $r$ is some radial distance.

\subsection{A Simple Model for the Timescale for Ambipolar Diffusion in the Presence of CO-coated Grains}

Initially the detailed density structure of the prestellar cloud is ignored, and average values of the magnetic field, the ion density, and the neutral density are used. The standard approximation is made that $d / d r B^{2} \sim B_{0}^{2} / r_{m}$, where $r_{\mathrm{m}}$ is the outer radius of the cloud and $B_{0}$ is the average magnetic field initially present throughout the prestellar core. It then follows 
(Flower \& Pineau des Forêts 2003; Draine 2011) that

$$
\tau=\left(2 \mu_{0} / B_{0}^{2}\right) r_{m}^{2}\left\langle n_{i}\right\rangle\left\langle n_{\mathrm{H}}\right\rangle k_{\mathrm{IM}} \gamma m_{\mathrm{H}}
$$

where $\left\langle n_{\mathrm{H}}\right\rangle$ and $\left\langle n_{\mathrm{i}}\right\rangle$ are the average volume densities of neutrals and ions within the core, $k_{\mathrm{IM}}$ is the momentum transfer rate coefficient for ion-neutral collisions, and $m_{\mathrm{H}}$ is the mass of the hydrogen atom. The collision partner of interest is $\mathrm{H}_{2}$. He, at $10 \%$ by number of $\mathrm{H}_{2}$, with a polarizability 3.7 times smaller, contributes $<3 \%$ to $k_{\mathrm{IM}}$ and is ignored here. The ions involved are $\mathrm{H}^{+}$and $\mathrm{H}_{3}^{+}$, and $\gamma$ is a weighted average of $m_{\text {ion }} m_{\mathrm{H} 2} /$ $\left(m_{\text {ion }}+m_{\mathrm{H} 2}\right)$. The proportion of $\mathrm{H}^{+}$to $\mathrm{H}_{3}^{+}$as a function of density (Walmsley et al. 2004) yields $\gamma=0.73-0.77$ over a wide range from $\left\langle n_{\mathrm{H}}\right\rangle=3.5 \times 10^{10}$ to $2 \times 10^{11} \mathrm{~m}^{-3}$. Using the same weighting, the effective momentum transfer rate coefficient becomes a weighted average of those for $\mathrm{H}^{+}$and $\mathrm{H}_{3}^{+}$, yielding an effective rate coefficient of $k_{\mathrm{IM}}$ varying between $2.96 \times 10^{-15}$ and $2.92 \times 10^{-15} \mathrm{~m}^{3} \mathrm{~s}^{-1}$. The product $k_{\mathrm{IM}} \gamma$ changes by only $3.5 \%$ over the density range mentioned and is treated as invariant with density throughout and equal to $2.24 \times 10^{-15} \mathrm{~m}^{3} \mathrm{~s}^{-1}$. The value of $B_{0}$ used here is given by the empirical relationship $B_{0}=1.549 \times 10^{-15} n_{n}^{0.65} \mathrm{~T}$ reported in Crutcher et al. (2010) and Crutcher (2012).

Equation (2) now allows an estimate of the time required to expel the magnetic field from a prestellar core. A value of $r_{\mathrm{m}}$ is taken to be $250^{\prime \prime}$ or $5.61 \times 10^{15} \mathrm{~m}$, from observational data for B68 (Nielbock et al. 2012), continuing to use characteristics of this object as typical, while noting the considerable variability between cores (Enoch et al. 2008). An average value of density $\left\langle n_{\mathrm{H}}\right\rangle$ may be obtained from the relation (Draine 2011) $r_{m}=2.39 \times 10^{24} n_{\mathrm{H}}^{-0.81} \mathrm{~m}$ yielding $\left\langle n_{\mathrm{H}}\right\rangle=4.46 \times 10^{10} \mathrm{~m}^{-3}$, where the values of $r_{\mathrm{m}}$ and $\left\langle n_{\mathrm{H}}\right\rangle$ are consistent with a total mass of the B68 avatar of $3.1 M_{\odot}$ (Nielbock et al. 2012). This yields $B_{0}=1.29 \times 10^{-8} \mathrm{~T}$.

Using $\quad n_{\mathrm{i}}=\alpha \chi\left\langle n_{\mathrm{H}}\right\rangle=6.7 \times 10^{-3} \chi\left\langle n_{\mathrm{H}}\right\rangle^{1 / 2}$, where $\chi=1$ and $\chi=1 / q_{p}=0.182$ for the non-spontelectric and spontelectric cases, respectively, Equation (2) gives an ambipolar diffusion lifetime, $\tau$, of $4.77 \times 10^{21} \chi\left\langle n_{\mathrm{H}}\right\rangle^{-1.42}$. This yields $3.6 \times 10^{6} \mathrm{yr}$ for $\chi=1$, and $\tau_{\mathrm{s}}$, for the spontelectric case, becomes $6.5 \times 10^{5} \mathrm{yr}$, for $\chi=0.182$. We note, however, that these values of ambipolar diffusion times rely on the idealized power-law relationships between $r_{m}, B_{0}$, and $n_{\mathrm{H}}$, whose accuracy may be disputed (Draine 2011). For example, recent work of Tritsis et al. (2015) suggests that for lowerdensity, nonspherical clouds, $B_{0}$ might be better written as proportional to $n_{n}^{0.5}$, and Li et al. (2015) suggest $n_{n}^{0.4}$ in NGC 6334. ${ }^{5}$ An alternative formulation (Flower \& Pineau des Forêts 2003; Walmsley et al. 2004) of $\tau$, which does not require the use of these power laws, save in the correction term, $E_{\mathrm{th}} / E_{B}$, small for pristine B68, is given by Equation (3):

$$
\tau=\left(2 \pi G m_{\mathrm{H}}\right)^{-1}\left(n_{\mathrm{i}} / n_{n}\right) k_{\mathrm{IM}} \gamma\left(1+E_{\mathrm{th}} / E_{\mathrm{B}}\right)
$$

where $E_{\mathrm{th}}$ is the thermal energy and $E_{\mathrm{B}}$ the initial magnetic energy per unit volume, that is, with $B_{0}=1.29 \times 10^{-8} \mathrm{~T}$. The ratio $E_{\mathrm{th}} / E_{B}$ is $\sim 0.07$ for the pristine B68 avatar. Equation (3)

\footnotetext{
5 Li et al. suggest a field of $B_{0} \propto n_{n}^{0.4}$ for NGC 6334 (see text). Transposed to B68, this corresponds to $B_{0}=7.4 \times 10^{-8} \mathrm{~T}$. This field is a factor of 5.7 times greater than the value based on Crutcher et al. (2010) and Crutcher (2012). This larger field yields an estimate of $\tau$ that is 32.5 times smaller than that calculated here from Equation (2), introducing, for example, a major discrepancy between Equations (2) and (3).
}

is, however, based on the assumption that material is in virial equilibrium, thereby establishing a relationship between the lower limit for $r_{m}$, the density of the medium, and the thermal and magnetic energy. The assumption of virialization cannot be generalized but may be appropriate to B68 (Nielbock et al. 2012; Roy et al. 2014). Equation (3) gives $\tau \sim 4.5 \%$ smaller than the value given by Equation (2). This agreement justifies use of the relationship, $\tau \propto\left\langle n_{\mathrm{H}}\right\rangle^{-1.42}$, derived above and in Draine (2011), which is used henceforth.

On the basis of Equations (2) and (3) a prestellar core of central density $\lesssim 5 \times 10^{11} \mathrm{H} \mathrm{m}^{-3}$ has a high probability of suffering disruption by SNRs before expulsion of the magnetic field in the absence of the spontelectric effect of solid CO. In its presence, the lifetime for expulsion of the magnetic field of typically $\sim 5 \times 10^{5} \mathrm{yr}$ falls well outside this disruption zone and within the broad range for the ages of prestellar cores derived from observations, mentioned in the opening paragraph of the current work. It is now clear how magnetically supercritical cores can form, and results have resolved problems of (i) $\tau$ matching observational lifetimes of prestellar cores, (ii) $\tau>\tau_{\mathrm{SNR}}$, and (iii) a route to magnetic supercriticality, raised in the introduction.

\subsection{Inclusion of Explicit Density Variation within the Core}

The density structure of the core, that is, the variation of $n_{\mathrm{H}}$ with $r$, is now included in the analysis. This is a useful extension since at small $r$, that is, at higher densities, the magnetic field escapes the core more rapidly and at lower density, at large $r$, less rapidly. The two effects, while working against each other, do not cancel. In order to include this behavior, we use the Plummer density distribution (e.g., Whitworth \& Ward-Thompson 2001; Nielbock et al. 2012; Lippok 2014),

$$
n_{\mathrm{H}}(r)=n_{0} /\left[1+\left(r / r_{0}\right)^{\eta / 2}\right]^{2}
$$

where $n_{0}$ is the central density of the cloud, $r_{0}$ is a parameter adjusted to fit observational data, and $\eta$ is conventionally taken to be equal to 4. Equation (4) is taken from hereon to be a universal representation of the density distribution of a prestellar core. Note that Equation (4) is not based on virialization (Dejonghe 1987). Using the relation $\tau \propto\left\langle n_{\mathrm{H}}\right\rangle^{-1.42}$ and incorporating the Plummer model of Equation (4) into this relation,

$$
\begin{aligned}
\tau= & \beta \chi \int_{r=0}^{r=r_{m}}\left[\frac{n_{0}}{\left(1+\left(r / r_{0}\right)^{2}\right)^{\eta / 2}}\right]^{-1.42} d r \\
& +5.58 \times 10^{15} \lambda /\left\langle n_{\mathrm{H}}\right\rangle
\end{aligned}
$$

where $\tau$ is in years, $\chi=1$ and $\lambda=0$ for the non-spontelectric case, and $\chi=\left(q_{p}\right)^{-1}=0.182$, as before, and $\lambda=1$ for the spontelectric case. The value of $\beta$ is found to be $6.71 \times 10^{3}$, using the adopted value of $k_{\mathrm{IM}} \gamma$ and relationships given above between $r_{m}, B$, and $n_{i}$ with $\left\langle n_{\mathrm{H}}\right\rangle$. The second and smaller term in Equation (5) takes into account the deposition time of $\mathrm{CO}$, under the assumption that the spontelectric effect requires 4-5 ML to initiate (Lasne et al. 2015). 


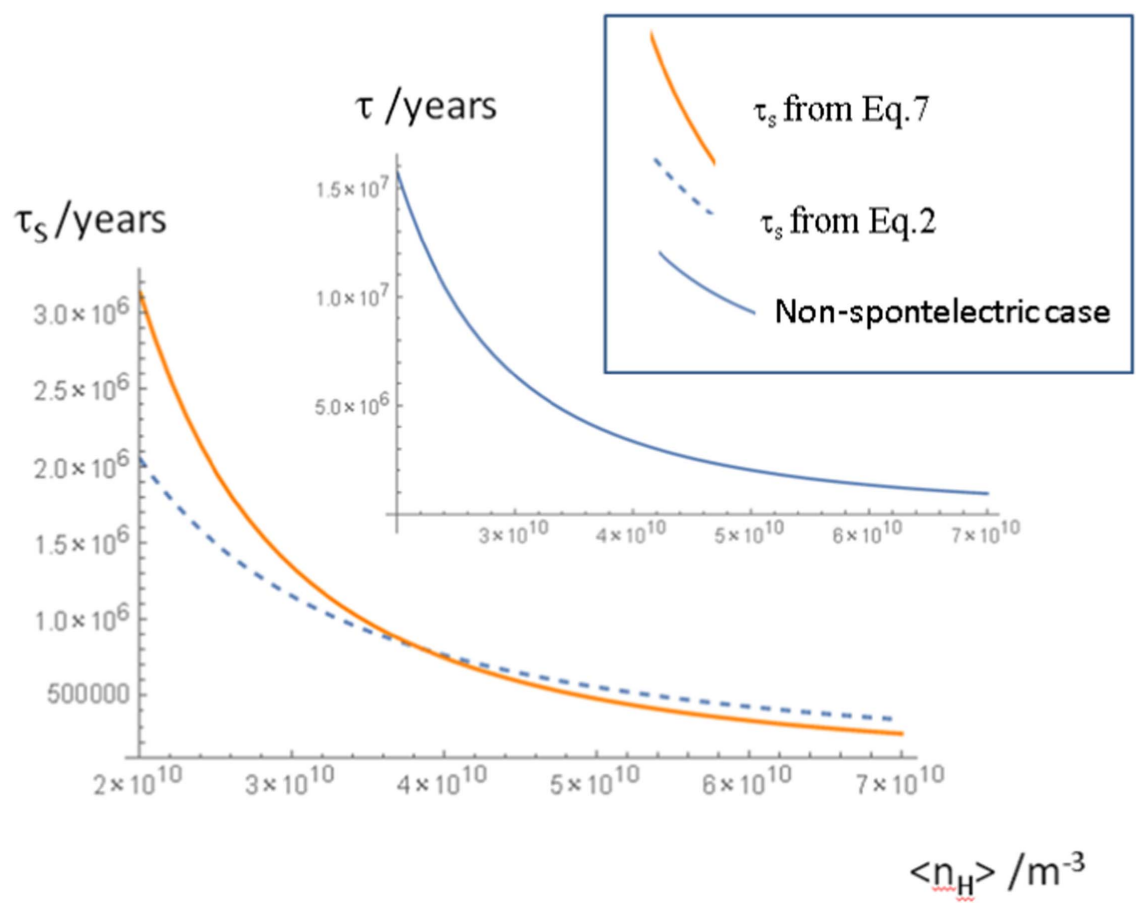

Figure 1. Ambipolar diffusion lifetime, $\tau_{\mathrm{s}}$, required to expel the magnetic field completely from a prestellar core as a function of the average number density of $\mathrm{H}$ atoms, $\left\langle n_{\mathrm{H}}\right\rangle$, in the presence of the spontelectric effect (Rosu-Finsen et al. 2016) in solid CO, computed using Equation (7) (solid orange curve). The values of $\left\langle n_{\mathrm{H}}\right\rangle$ are taken from the observationally based relation $r_{m}=2.39 \times 10^{24}\left\langle n_{\mathrm{H}}\right\rangle^{-0.81} \mathrm{~m}$, where $r_{m}$ is the radial extent of any core. Results are computed using a Plummer density distribution (Equation (4)) within the cloud, where parameters have been determined by comparison with the prestellar core B68. The dashed curve shows the variation $\tau_{\mathrm{s}}=8.67 \times 10^{20} n_{\mathrm{H}}^{-1.42} \mathrm{yr}$ for the model in which the density structure of the core is ignored (Equation (2)). The inset shows results for the non-spontelectric case. Random errors in estimates of $\tau$ and $\tau_{\mathrm{s}}$ are $\pm 6 \%$ in the magnetic field, $11 \%$ in $\left\langle n_{\mathrm{H}}\right\rangle, 8 \%$ in $r_{m}, 6 \%$ in $n_{i}$, and $7 \%$ in $\chi$, giving an overall error of $\pm 20 \%$. Systematic errors stem very largely from the power laws relating $B$ or $r_{\mathrm{m}}$ with $\left\langle n_{\mathrm{H}}\right\rangle$.

Equation (5) then yields

$$
\begin{aligned}
\tau & =6.71 \times 10^{3} \\
& \times \chi \frac{r_{m}\left(1+r_{m}^{2} / r_{0}^{2}\right)^{-0.71 \eta}{ }_{2} F_{1}\left(0.5,-0.71 \eta ; 1.5 ;-r_{m}^{2} / r_{0}^{2}\right)}{\left[n_{0}\left(1+r_{m}^{2} / r_{0}^{2}\right)^{\eta / 2}\right]^{1.42}} \\
& +5.58 \times 10^{15} \lambda /\left\langle n_{\mathrm{H}}\right\rangle
\end{aligned}
$$

where ${ }_{2} F_{1}(\ldots)$ is Gauss's hypergeometric function. Using Equation (4) to fit to a cloud mass for B68 of $3.1 M_{\odot}$, it may be shown that $n_{0} \sim 7.62\left\langle n_{\mathrm{H}}\right\rangle$ and $r_{0} \sim 0.2 r_{m}$, which are general relationships. Evaluation of Equation (6) yields

$$
\begin{aligned}
\tau & =\frac{1.47 \times 10^{30}}{\left\langle n_{\mathrm{H}}\right\rangle^{2.23}} \\
\text { and } \tau_{s} & =\frac{2.67 \times 10^{29}}{\left\langle n_{\mathrm{H}}\right\rangle^{2.23}}+\frac{5.58 \times 10^{15}}{\left\langle n_{\mathrm{H}}\right\rangle}
\end{aligned}
$$

where $\tau_{\mathrm{s}}$, as throughout, includes the spontelectric effect. As before, $\left\langle n_{\mathrm{H}}\right\rangle=4.46 \times 10^{10} \mathrm{~m}^{-3}$, which now gives $\tau=2.6 \times 10^{6} \mathrm{yr}$ and $\tau_{\mathrm{s}}=4.8 \times 10^{5}+1.25 \times 10^{5} \mathrm{yr}=$ $6.05 \times 10^{5} \mathrm{yr}$. The variation of $\tau_{\mathrm{s}}$ with $\left\langle n_{\mathrm{H}}\right\rangle$ is shown in Figure 1. Also shown is the variation of $\tau_{\mathrm{s}}$ without inclusion of density variation, illustrating that the slower expulsion associated with the low-density tail outweighs the more rapid expulsion at high density for $\left\langle n_{\mathrm{H}}\right\rangle \lesssim 4 \times 10^{10} \mathrm{H} \mathrm{m}^{-3}$, and vice versa for higher densities. The inset shows the variation of the non-spontelectric value $\tau \propto\left\langle n_{\mathrm{H}}\right\rangle^{-2.23}$ for comparison. Random errors in derived values of $\tau$ and $\tau_{\mathrm{s}}$ are given in the caption to Figure 1.
The above analysis can be used to calculate a density threshold for star formation. This is performed by obtaining the value of the average density, $\left\langle n_{\mathrm{H}}\right\rangle \mathrm{m}^{-3}$, such that $\tau \leqslant \tau_{\mathrm{SNR}}$ using Equation (7). Thus, if we require, say, that $\tau_{\mathrm{s}}$ is $\leqslant 2.5 \pm 0.5 \mathrm{Myr}$, to avoid disruption by SNRs, then $\left\langle n_{\mathrm{H}}\right\rangle$ must be $\geqslant(2.2 \pm 0.2) \times 10^{10} \mathrm{H} \mathrm{m}^{-3}$, that is, $\geqslant(1.1 \pm 0.1)$ $\times 10^{4} \mathrm{H}_{2} \mathrm{~cm}^{-3}$. The corresponding (non-spontelectric) $\tau$ is $\sim 13 \mathrm{Myr}$ at this number density. The density for the spontelectric case agrees well with a value for the threshold for star formation, derived from observations (Lada et al. 2010; Parmentier et al. 2011) of molecular cloud complexes, of $\sim 10^{4} \mathrm{H}_{2} \mathrm{~cm}^{-3}$, given that the greater part of the magnetic field must be expelled for gravitational collapse to be feasible.

\section{CONCLUDING COMMENTS}

The current work contributes to a new and global understanding of the initial collapse of prestellar cores to form stars. Quantitative uncertainties in our analysis arise principally from two sources: the considerable variability in estimates of the age of prestellar cores $\left(10^{5}\right.$ to $\left.>3 \times 10^{6} \mathrm{yr}\right)$ and the uncertainty in the relationship between the value of the pristine magnetic field and the local core density. These uncertainties do not in themselves undermine the generality of the results presented here, but suggest that, for the full spectrum of core conditions encountered in the Galaxy and in external galaxies, the spontelectric effect may range from highly influential to of little impact for the fate of prestellar cores. Notwithstanding these reservations, a link has been established between the disappearance of $\mathrm{CO}$ from the gas phase in cold quiescent regions and the degree of ionization of the ISM. This link is shown to facilitate the expulsion of the magnetic field by 
reduction of the degree of ionization in the core, hastening the natural mechanism for the creation of magnetically supercritical cores. Cores are demonstrated to become magnetically supercritical in a time that is now several times less than the average time between disruptions by $\mathrm{SNe}$, depending on the core density (Figure 1).

This finding implies a greater rate of star formation in galaxies in general, with more candidate cores achieving gravitational collapse. The implications of the result for galaxy formation have yet to be considered but constitute an interesting area of future work. The spontelectric effect of solid $\mathrm{CO}$ in reducing the degree of ionization may also have some general influence in the field of astrochemistry and in the nature of $\mathrm{C}$-type shocks entering quiescent regions.

\section{REFERENCES}

Balog, R., Cicman, P., Jones, N., \& Field, D. 2009, PhRvL, 102, 2 Banerjee, R., \& Körtgen, B. 2015, arXiv:1509.03436v1

Bergin, E. A., \& Tafalla, M. 2007, ARA\&A, 45, 339

Brünken, S., Sipalä, O., Chambers, E. T., et al. 2014, Natur, 516, 219

Caselli, P. 2011, in IAU Symp. 280, The Molecular Universe, ed. J. Cernicharo \& R. Bachiller (Cambridge: Cambridge Univ. Press), 19

Cassidy, A., Plekan, O., Balog, R., Jones, N. C., \& Field, D. 2012, PCCP, 15,108

Cassidy, A., Plekan, O., Dunger, J., et al. 2014, PCCP, 16, 23843

Collings, M. P., Dever, J. W., \& McCoustra, M. R. S. 2014, PCCP, 16, 3479

Crutcher, R. M. 2012, ARA\&A, 50, 29

Crutcher, R. M., Wandelt, B., Heiles, C., Falgarone, E., \& Troland, T. H. 2010, ApJ, 725, 466

Dejonghe, H. 1987, MNRAS, 224, 13

Diehl, R., Halloin, H., Kretschmer, K., et al. 2006, Natur, 439, 45

Draine, B. T. 2011, Physics of the Interstellar and Intergalactic Medium (Princeton, NJ: Princeton Univ. Press)

Draine, B. T., \& Sutin, B. 1987, ApJ, 320, 803

Enoch, M. L., Evans, N. J., Sargent, A. I., et al. 2008, ApJ, 684, 1240

Evans, N. J., Dunham, M. M., Joergensen, J. K., et al. 2009, ApJSS, 181, 321

Field, D. 2000, A\&A, 362, 774
Field, D., Plekan, O., Cassidy, A., et al. 2013, IRPC, 32, 345

Flower, D. R., \& Pineau des Forêts, G. 2003, MNRAS, 341, 1272

Ford, A. B., \& Shirley, Y. L. 2011, ApJ, 728, 144

Kandori, R., Tanura, M., Tatematsu, K., et al. 2009, in IAU Symp. 259 , Planets, to Stars and Galaxies, ed. K. G. Strassmeier, A. G. Kosovichev, \& J. E. Beckman (Cambridge: Cambridge Univ. Press), 107

Könyves, V., André, P., Menshikov, A., et al. 2015, A\&A, 584, A91

Korolev, V. V., Vasiliev, E. O., Kovalenko, I. G., \& Shchekinov, Y. A. 2015, arXiv: $1506.09038 \mathrm{v} 1$

Lada, C., Lombardi, M., \& Alves, J. F. 2010, ApJ, 724, 687

Lasne, J., Rosu-Finsen, A., Cassidy, A., McCoustra, M. R. S., \& Field, D. 2015, PCCP, 17, 31077

Li, H. B., Yuen, K. H., Otto, F., et al. 2015, Natur, 520, 518

Lippok, N. 2014, Ph.D.Thesis, http://archiv.ub.uni-heidelberg.de/ volltextserver/16221/1/thesis_Lippok.pdf

Maret, S., \& Bergin, E. A. 2007, ApJ, 664, 956

Maret, S., Bergin, E. A., \& Tafalla 2013, A\&A, 559, A53

Mathis, J. S., Rumpl, W., \& Nordsieck, K. H. 1977, ApJ, 217, 425

Nielbock, M., Launhardt, R., Steinacker, J., et al. 2012, A\&A, 547, A11

Pagani, L., Bourgoin, A., \& Lique, F. 2012, A\&A, 548, L4

Pagani, L., Lesaffre, P., Jorfi, M., et al. 2013, A\&A, 551, A38

Parise, B., Belloche, A., Du, F., Gusten, R., \& Menten, K. M. 2011, A\&A, 526, A31

Parmentier, G., Kaufmann, J., Pillai, T., \& Menten, K. M. 2011, MNRAS, 416, 783

Plekan, O., Cassidy, A., Balog, R., Jones, N. C., \& Field, D. 2011, PCCP, 13, 21035

Rosu-Finsen, A., Lasne, J., Cassidy, A., McCoustra, M. R. S., \& Field, D. 2016, PCCP, 18, 5159

Roy, A., André, P. h., Palmeirim, P., et al. 2014, A\&A, 562, A138

Sadavoy, S. I., Shirley, Y., Di Francesco, J., et al. 2015, arXiv:1504.05206v1

Tielens, A. G. G. M. 2006, The Physics and Chemistry of the Interstellar Medium (Cambridge: Cambridge Univ. Press)

Tristis, A., Tassis, K., \& Willacy, K. 2016, MNRAS, 458, 789

Umebayashi, T., \& Nakano, T. 1980, PASJ, 32, 405

Walmsley, C. M., Flower, D. R., \& Pineau des Forêts, G. 2004, A\&A, 418, 1035

Ward-Thompson, D., André, P., Crutcher, R., et al. 2007, in Protostars and Planets V, ed. B. Reipurth, D. Jewitt, \& K. Keil (Tucson, AZ: Univ. Arizona Press), 33

Whitworth, A. P., \& Ward-Thompson, D. 2001, ApJ, 547, 317 